\title{
Synthesis and Characterization of Hyaluronic Acid- $\alpha$-Cyclodextrin Conjugate as the Potential Carrier of PEGylated Drugs
}

\author{
Maharajan Sivasubramanian ${ }^{1}$ and Jae Hyung Park ${ }^{1,2 \uparrow}$ \\ ${ }^{1}$ Departments of Advanced Polymer and Chemical Engineering, Kyung Hee University, Gyeonggi-do 446-701, South Korea \\ ${ }^{2}$ Department of Life and Nanopharmaceutical Sciences, Kyung Hee University, Seoul 130-701, South Korea \\ (Received July 1, 2010 • Revised August 9, 2010 • Accepted August 10, 2010)
}

\begin{abstract}
The hyaluronic acid (HA) conjugate bearing $\alpha$-cyclodextrin $(\alpha-\mathrm{CD})$ was synthesized as the potential carrier of poly(ethylene glycol) (PEG)-drug conjugates. The HA conjugate was prepared by the reaction between the carboxylic acid of HA and the primary amine of $\alpha$-CD in the presence of 1-ethyl-3-(3-dimethylaminopropyl) carbodiimide and 1hydroxybenzotriazole. The chemical structure of the conjugate was confirmed using ${ }^{1} \mathrm{H}$ NMR and FT-IR spectroscopy. The conjugate could form nano-sized particles in the presence of PEG by forming the inclusion complexes between $\alpha$-CD at the backbone of HA, which was demonstrated using electrophoretic light scattering and field emission transmission electron microscopy. It is anticipated that this novel kind of nanoparticles can serve as a useful delivery system for PEGylated drugs.
\end{abstract}

Key words - Hyaluronic acid, $\alpha$-cyclodextrin, PEG, nanoparticles.

Cyclodextrins (CDs), composed of $D$-glucose units linked through $\alpha-(1 \rightarrow 4)$ glycosidic bonds, are water-soluble cyclic oligosaccharides with truncated cone shapes. They have hydrophobic inner cavities and hydrophilic outer surfaces, which enables them to form inclusion complexes with a variety of hydrophobic molecules. This unique feature of the CDs has allowed development of pharmaceutical formulations to improve physicochemical properties of the hydrophobic drugs such as solubility, stability, and bioavailability (Charlot et al., 2006; Szejtli., 1998; Uekama et al., 1998; Loftsson and Brewster., 1996). In addition to hydrophobic small molecules, CDs are known to form inclusion complexes with various polymers such as poly(ethylene glycol) (PEG) and poly(propylene glycol) (PPG). The guest molecules to form the complexes are determined by cavity sizes of the CDs (Harada., 1996). For example, $\alpha-C D$ can form the inclusion complexes with PEG, whereas $\beta-C D$ can form complexes with PPG. The PEG chain can easily pass through the $\alpha-C D$ cavity, followed by the physical interaction to form the stable complex. On the other hand, PPG can form the complex with $\beta-C D$, possessing the cavity larger than $\alpha-C D$, because PPG has the bulky methyl group at the polymeric backbone.

PEG, approved by food and drug administration (FDA) in USA, has been widely used for various biomedical applications such as drug delivery and tissue engineering. PEGylation,

†Corresponding Author:

Tel : +82-31-201-3256, E-mail : jaehyung@khu.ac.kr

DOI : 10.4333/KPS.2010.40.4.219 a technology based on the covalent attachment of PEG to therapeutic drugs including proteins, has been demonstrated to improve pharmacokinetics of the drugs, enhance stability, increase solubility, reduce immunogenicity, and extend biological half-lives by reducing renal clearance (Veronese and Paust., 2005; Duncan., 2003). Hyaluronic acid (HA), consisting of repeating units of $N$-acetyl- $D$-glucosamine and $D$-glucuronic acid, is a linear polysaccharide found in the extracellular matrix and synovial fluid of the joint. Owing to its intrinsic properties such as viscoelasticity, biodegradability, and biocompatibility, HA has been extensively used for tissue engineering and drug delivery (Lee et al., 2008; Yadav et al., 2008., Lapcík et al., 1998). For example, HA can bind to the receptor CD44, over-expressed in various cancer cells, which allows its use as the targeting ligand for drug delivery (Götte and Yip., 2006; Eliaz and Szoka., 2001; Luo and Prestwich., 1999). Since HA has the carboxylic acid at its repeating unit, its physicochemical properties can be readily controlled via the chemical conjugation using the functionally reactive compounds.

In this study, the HA conjugate bearing the $\alpha-C D$ was synthesized as the potential carrier of PEGylated drugs. Since PEG forms inclusion complexes with $\alpha-C D$ at the backbone of $\mathrm{HA}$, it is expected that the conjugate can improve stability of the drug and allow its sustained release from the complexes. The conjugate was obtained by preparation of the $\alpha-C D$ possessing the primary amino group, followed by the coupling reaction with HA. The chemical structure of the conjugate was confirmed by ${ }^{1} \mathrm{H}$ NMR and FT-IR spectroscopy. The complex formation of the conjugate with PEG was demonstrated using 
electrophoretic light scattering and field emission transmission electron microscopy.

\section{Experimental}

\section{Materials}

HA (MW $=300 \mathrm{~K})$ was obtained from Lifecore Biomedical (MN, USA). $\alpha-\mathrm{CD}$ and 1-hydroxy benzotriazole (HOBt) were purchased from Fluka (Steinheim, Switzerland) and TCI (Tokyo, Japan), respectively. 1-Ethyl-3(3-dimethylaminopropyl) carbodiimide hydrochloride (EDC), PEG (MW=10 K), and all other chemicals were purchased from Sigma-Aldrich (St. Louis, MO, USA) and used without further purification.

\section{Methods}

Synthesis of 6-amino- $\alpha$-cyclodextrin

6 -Amino- $\alpha$-cyclodextrin was prepared with slight modification of the report published previously (Hamasaki et al., 1993). In brief, $\alpha$-CD (10 g) and $p$-toluenesulfonyl chloride $(10 \mathrm{~g})$ were dissolved in pyridine $(750 \mathrm{~mL})$ at $5^{\circ} \mathrm{C}$. The reaction mixture was stirred for $5 \mathrm{~h}$ at room temperature and was precipitated in acetone, followed by purification in an activated charcoal column to obtain monotosyl $\alpha-C D(2.3 \mathrm{~g})$. The product was suspended in distilled water $(50 \mathrm{~mL})$ containing sodium azide $(3 \mathrm{~g})$, which was then stirred for $5 \mathrm{~h}$ at $80^{\circ} \mathrm{C}$. The resulting mixture was precipitated with acetone and dried for 2 days at room temperature under vacuum to obtain azide-bearing $\alpha-C D$. Thereafter, it was dissolved in DMF $(20 \mathrm{~mL})$ containing triphenyl phosphine $(1 \mathrm{~g})$, to which concentrated $\mathrm{NH}_{3}$ (aq) $(7 \mathrm{~mL})$ was added. The solution was stirred at room temperature for $4 \mathrm{~h}$, precipitated in acetone, and purified by the ion exchange chromatography on a Sephadex column eluted with $1 N \mathrm{NH}_{3}$ (aq) to obtain 6-amino- $\alpha$-cyclodextrin $(1.4 \mathrm{~g})$.

\section{Synthesis of $H A-\alpha-C D$ Conjugate.}

HA (100 mg) was dissolved in distilled water at a concentration of $3 \mathrm{mg} / \mathrm{mL}$, to which an excess amount of 6-amino$\alpha$-cyclodextrin $(3.6 \mathrm{~g}, 3.75 \mathrm{mmol})$ was added. The $\mathrm{pH}$ of the solution was adjusted to 6.8 with $0.1 \mathrm{MNaOH}$ and $0.1 \mathrm{M} \mathrm{HCl}$. EDC (192 mg, $1 \mathrm{mmol})$ and HOBt (135 mg, $1 \mathrm{mmol})$, dissolved in $\mathrm{DMSO} / \mathrm{H}_{2} \mathrm{O}(1: 1,1 \mathrm{~mL})$, was added to the solution and the reaction was allowed to proceed overnight. After the reaction, the $\mathrm{pH}$ of the solution was adjusted to 7.0 with $0.1 \mathrm{M}$ $\mathrm{NaOH}$ and was extensively dialyzed (MW cutoff 12-14000) against distilled water, followed by lyophilization.

\section{Characterization}

The FT-IR spectrum of the conjugate was obtained using a
Bruker 66V FT-IR spectrophotometer (Ettlingen, Germany) at a resolution of $4 \mathrm{~cm}^{-1}$ with 100 scans. ${ }^{1} \mathrm{H}$ NMR spectrum was obtained using the NMR spectrometer (JEOL, JNM-AL300) operating at $300 \mathrm{MHz}$, in which the sample was dissolved in $\mathrm{D}_{2} \mathrm{O}$ at a polymer concentration of $1 \mathrm{mg} / \mathrm{mL}$.

The nanoparticles were prepared by mixing the HA- $\alpha-C D$ conjugate with PEG in distilled water. The solution was further sonicated using a probe type sonifier (VCX-750, Sonics \& Materials) at $90 \mathrm{~W}$ in which the pulse was turned off for $2 \mathrm{~s}$ with an interval of $5 \mathrm{~s}$. The solution was passed through a syringe type membrane filter $(0.85 \mu \mathrm{m}$, millipore). The particle size and size distribution of the inclusion complex were determined by an electrophoretic light scattering (ELS, Photal, ELSZ-2), in which the scattered light was measured at $90^{\circ}$. The morphology of the nanoparticles was observed using field emission transmission electron microscope (Fe-TEM), operated at an accelerating voltage of $200 \mathrm{keV}$. The sample was prepared at a concentration of $1 \mathrm{mg} / \mathrm{mL}$ in distilled water, placed in the copper grid, and dried under vacuum.

\section{Results and Discussion}

In recent years, $\alpha-C D$ has been considered as the potential carrier of PEGylated drugs for their sustained release in the biological environment because $\alpha-C D$ is capable of forming inclusion complex with PEG which is insoluble in aqueous media (Harada and Kamachi., 1990). However, the inclusion complex between $\alpha-\mathrm{CD}$ and PEG, by the hydrogen bonding, is not sufficiently stable in the biological fluids. Therefore, the PEGylated drugs were released from the complex within several hours, although this formulation showed better pharmacokinetics than free PEGylated drugs (Higashi et al., 2009; Higashi et al., 2007). In this study, we aimed to develop $\alpha-C D-$ bearing polymer conjugates to form nano-sized inclusion complexes with PEGylated drugs, which may allow sustained release of the PEGylated drugs by hydrogen bonding as well as entanglements with the polymer chain. The overall synthetic route is shown in Figure 1.

\section{Synthesis and Characterization of HA- $\alpha-C D$ Conjugate}

6-Amino- $\alpha$-cyclodextrin is prepared by the three-step process. Since this $\alpha-C D$ derivative has the primary amino group, it can react with the carboxylic acid of HA. At the first step, $\alpha$-CD was monotosylated in the presence of $p$-toluenesulfonyl chloride, in which pyridine was used as a solvent. Pyridine forms complex with $\alpha-C D$ and favors mainly the formation of monotosylated derivative at low temperature. The multi-substituted $\alpha$-CDs were purified by using the column chroma- 

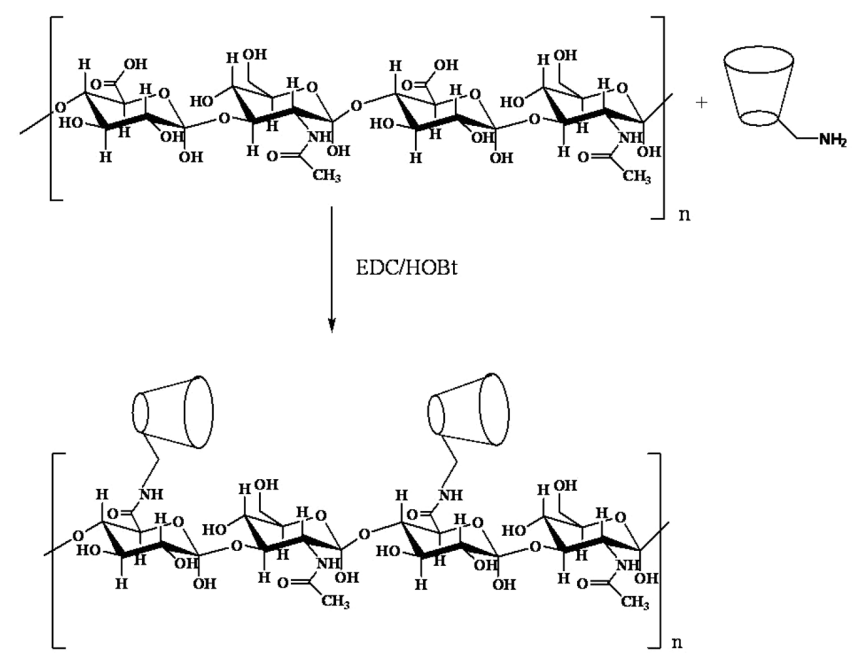

Figure 1. Synthesis of HA- $\alpha-C D$ conjugate.

tography. At the second step, the tosyl group of $\alpha-C D$ was substituted with the azide functional group, which was then reduced to the primary amino group via the Staudinger reaction at the final step. The ${ }^{1} \mathrm{H}$ NMR spectrum of 6 -amino- $\alpha$ cyclodextrin is shown in Figure 2.

6-Amino- $\alpha$-cyclodextrin was then chemically conjugated into the backbone of HA. For this reaction, the carboxylic acid of HA was activated in the presence of EDC and HOBt, which is a well-known procedure for preparing HA derivatives (Bulpitt and Aeschilmann., 1999). The reaction was performed at a $\mathrm{pH}$ of 6.8 for the effective coupling; otherwise, formation of the $N$-acylurea is favored, which will hinder the coupling between HA and 6-amino- $\alpha$-cyclodextrin.

The FT-IR spectra of HA and HA- $\alpha-C D$ is shown in Figure 3 . The HA- $\alpha-C D$ spectrum clearly showed the strong signal of $\mathrm{C}-\mathrm{O}$ stretching vibration band at 1150 and $1030 \mathrm{~cm}^{-1}$, denoting the presence of $\alpha-C D$, and the signal at $1620-1640 \mathrm{~cm}^{-1}$ for amide $\mathrm{C}=\mathrm{O}$ stretching. This result implies that $\alpha-\mathrm{CD}$ is conjugated into the HA via the formation of the amide bond.

The ${ }^{1} \mathrm{H}$ NMR spectrum of HA- $\alpha-C D$ conjugate is shown in

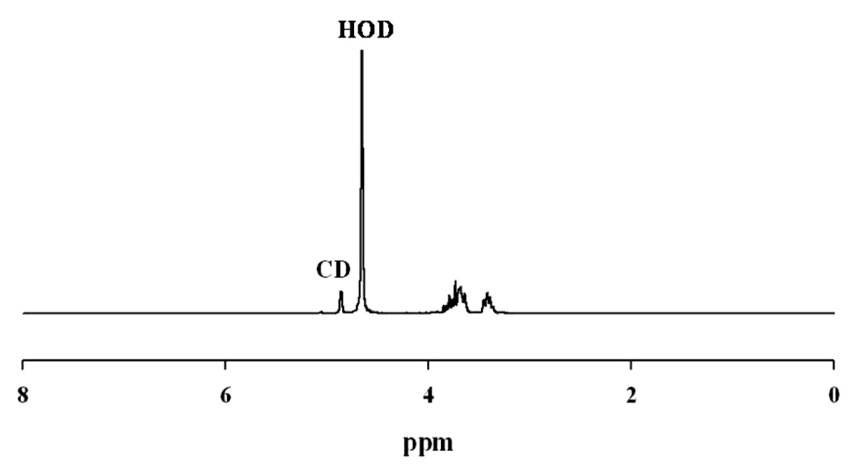

Figure 2. ${ }^{1} \mathrm{H}$ NMR spectrum of 6-amino- $\alpha$-cyclodextrin in $\mathrm{D}_{2} \mathrm{O}$.

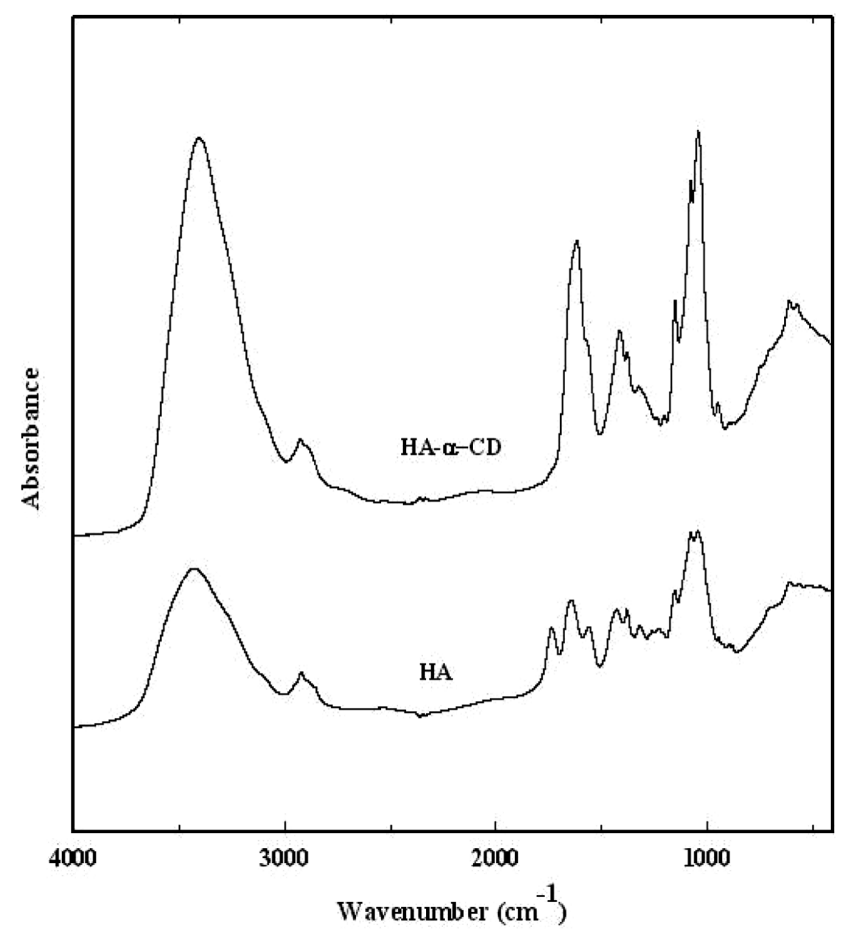

Figure 3. FT-IR spectrum of HA and $H A-\alpha-C D$ conjugate.

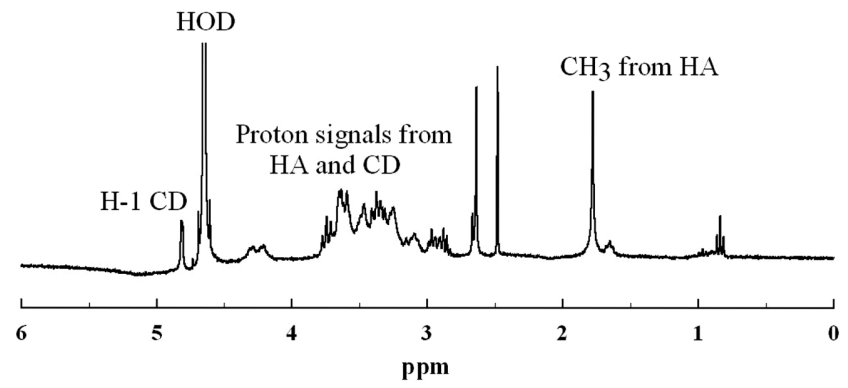

Figure 4. ${ }^{1} \mathrm{H}$ NMR spectrum of $\mathrm{HA}-\alpha-\mathrm{CD}$ conjugate in $\mathrm{D}_{2} \mathrm{O}$.

Figure 4. The characteristic peaks were observed at $\delta 4.8$ (anomeric protons of $\alpha-\mathrm{CD}, 4.42$ ( $H-1$ from $N$-acetyl glucosamine unit), 4.23 ( $H-1$ from glucuronic acid), 3.8-3.15 (protons of $\mathrm{HA}$ and $\alpha-\mathrm{CD})$, and $1.85\left(\mathrm{CH}_{3}\right.$ from acetamide of $\left.\mathrm{HA}\right)$. The degree of substitution of $\alpha-C D$ on the HA backbone was $10.3 \%$ which was calculated from the spectra by the integration ratio between the characteristic peak of the $\mathrm{N}$-acetyl group in $\mathrm{HA}\left(\delta=1.8 \mathrm{ppm}\left[3 \mathrm{H},-\mathrm{COCH}_{3}-\right]\right)$ and that of the anomeric protons in $\alpha-\mathrm{CD}(\delta=4.8 \mathrm{ppm},[6 \mathrm{H}])$.

\section{Characterization of Nanoparticles}

Since $\alpha-C D$ can form the inclusion complex with PEG, it can be expected that large aggregates or particles are formed in the presence of HA- $\alpha-C D$ and PEG. The large aggregates can be generated at the small amount of PEG which may play a role as the physical cross-linker between HA- $\alpha-C D$ conju- 


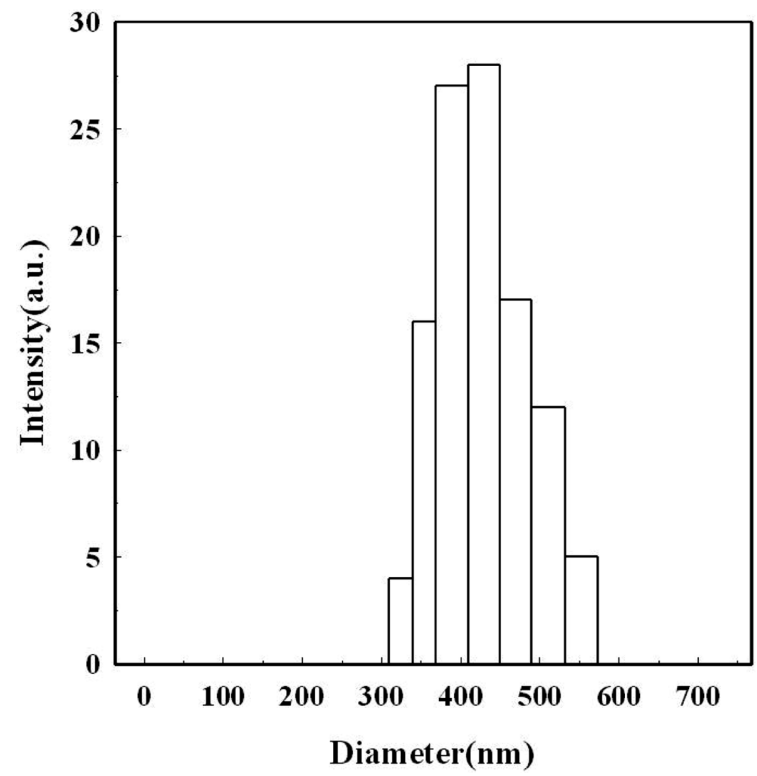

Figure 5. The particles size distribution of HA- $\alpha-C D-P E G$ nanoparticles.

gates. One the other hand, at the large amount of PEG, the particles can be obtained due to negligible inter-particular interactions. In this study, we aimed to make the nano-sized complexes which are useful for systemic administration of the drugs without surgical operation. Therefore, HA- $\alpha-C D$ was exposed to the excess amount of PEG $(20 \mathrm{wt} \%$ in distilled water) to investigate its potential forming nanoparticles with PEGylated drugs.

The formation of the nano-sized inclusion complex between HA- $\alpha-C D$ and PEG was demonstrated using the particle size measurement from ELS, as shown in Figure 5. Also, from the Fe-TEM image, it was found that the particles were spherical in shape (Figure 6). The size of nanoparticles ranged from 50 to $300 \mathrm{~nm}$. PEGylation of drugs has been developed for the
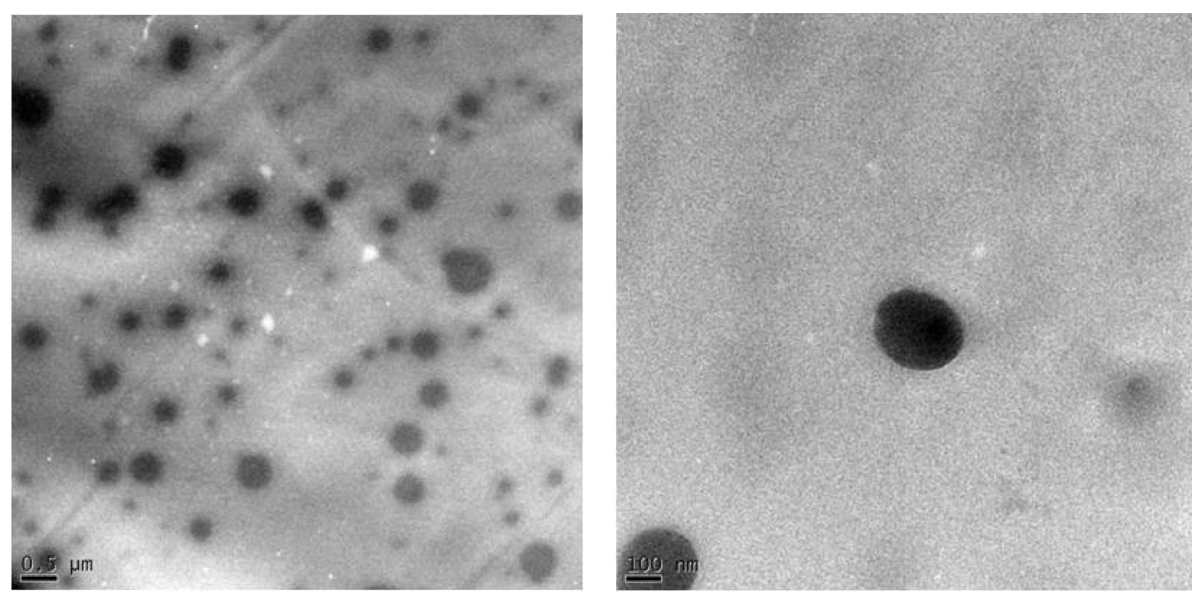

Figure 6. Fe-TEM image of HA- $\alpha$-CD-PEG nanoparticles.

systemic and prolonged circulation of drugs in vivo, due to the increase in molecular weight of the drugs. Recently, it has been suggested that $\alpha-C D$ has a potential as the carrier of PEGylated protein drugs by forming water-insoluble polypseudorotaxane (Higashi et al., 2008). The nanoparticular system, developed in this study, would receive much attention because the nano-sized particles can be used as an effective strategy in drug delivery especially for drug targeting to the specific site of action. Along this line of the research, the release behaviors of the PEGylated drugs from the inclusion complex and their biological activities are under investigation.

\section{Conclusion}

A novel HA- $\alpha-C D$ conjugate was synthesized by using 6amino- $\alpha$-cyclodextrin and HA in the presence of EDC and HOBt. The conjugate was able to form nanoparticles upon the addition of PEG, in which formation of the nanoparticle is attributed to the supramolecular complex formation by hydrogen bonding between $\alpha-C D$ at the backbone of the conjugate and PEG. This novel conjugate is of great interest as the potential carrier of PEGylated drugs.

\section{Acknowledgements}

This work was supported by the Ministry of Education, Science and Technology (Grant Numbers: 20090081871 and 20100015804).

\section{References}

Bulpitt, P., Aeschilmann, D., 1999. New strategy for chemical modification of hyaluronic acid: Preparation of functionalized 
derivatives and their use in the formation of novel biocompatible hydrogels. J. Biomed. Mater. Res. Part A. 47(2), 152169.

Charlot, A., Heyraud, A., Guenot, P., Rinaudo, M., Auzély-Velty, R., 2006. Controlled synthesis and inclusion ability of a hyaluronic acid derivative bearing $\beta$-cyclodextrin molecules. Biomacromolecules. 7(3), 907-913.

Duncan, R., 2003. The dawning era of polymer therapeutics. Nat. Rev. Drug Discov. 2(5), 347-360.

Eliaz, R.E., Szoka, F.C.Jr., 2001. Liposome-encapsulated doxorubicin targeted to CD44: A strategy to kill CD44-overexpressing tumor cells. Cancer Res. 61(6), 2592-2601.

Götte, M., Yip, G.W., 2006. Heparanase, hyaluronan, and CD44 in cancers: A breast carcinoma perspective. Cancer Res. 66: (21), 10233-10237.

Hamasaki, K., Ikeda, H., Nakamura, A., Ueno, A., Toda, F., Suzuki, I., Osa, T., 1993. Fluorescent sensors of molecular recognition. Modified cyclodextrins capable of exhibiting guestresponsive twisted intramolecular charge transfer fluorescence. J. Am. Chem. Soc. 115(12), 5035-5040.

Harada, A., 1996. Preparation and structures of supramolecules between cyclodextrins and polymers. Coord. Chem. Rev. 148, 115-133.

Harada, A., Kamachi, M., 1990. Complex formation between poly (ethylene glycol) and $\alpha$-cyclodextrin. Macromolecules. 23(10), 2821-2823.

Higashi, T., Hirayama, F., Misumi, S., Motoyama, K., Arima, H., Uekama, K., 2009. Polypseudorotaxane formation of randomly-pegylated insulin with cyclodextrins: slow release and resistance to enzymatic degradation. Chem. Pharm. Bull.
57(5), 541-544.

Higashi, T., Hirayama, F., Misumi, S., Arima, H., Uekama, K., 2008. Design and evaluation of polypseudorotaxanes of pegylated insulin with cyclodextrins as sustained release system. Biomaterials. 29(28), 3866-3871.

Higashi, T., Hirayama, F., Arima, H., Uekama, K., 2007. Polypseudorotaxanes of pegylated insulin with cyclodextrins: Application to sustained release system. Bioorg. Med. Chem. Lett. 17(7), 1871-1874.

Lapčík, L.Jr., Lapčík, L., Smedt, S.D., Demeester, J., Chabreček, P., 1998. Hyaluronan: preparation, structure, properties, and applications. Chem. Rev. 98(8), 2663-2684.

Lee, H., Lee, K., Park, T.G., 2008. Hyaluronic acid-paclitaxel conjugate micelles: Synthesis, characterization and antitumor activity. Bioconjugate Chem. 19(6), 1319-1325.

Loftsson, T., Brewster, M.E., 1996. Pharmaceutical applications of cyclodextrins. 1. Drug solubilization and stabilization. J. Pharm. Sci. 85(10), 1017-1025.

Luo, Y., Prestwich, G.D., 1999. Synthesis and selective cytotoxicity of a hyaluronic acid-antitumor bioconjugate. Bioconjugate Chem. 10(5), 755-763.

Szejtli, J., 1998. Introduction and general overview of cyclodextrin chemistry. Chem. Rev. 98(5), 1743-1754.

Uekama, K., Hirayama, F., Irie, T., 1998. Cyclodextrin drug carrier systems. Chem. Rev. 98(5), 2045-2076.

Veronese, F.M., Paust, G., 2005. Pegylation, successful approach to drug delivery. Drug Discov. Today. 10(21), 1451-1458.

Yadav, A.K., Mishra, P., Agarwal, G.P., 2008. An insight on hyaluronic acid in drug targeting and drug delivery. J. Drug Target. 16(2), 91-107. 